\title{
Study of Obsessive Compulsive Symptoms in Patients with Schizophrenia
}

\author{
Dr. Dhun Patel, Dr. Lakhan Kataria, Dr. Suhani Desai, Dr. Kinnari Trivedi \\ Department of Psychiatry, SBKS Medical Institute and Research Centre.
}

DOI: $10.29322 /$ IJSRP.10.03.2020.p9942

http://dx.doi.org/10.29322/IJSRP.10.03.2020.p9942

\begin{abstract}
-
Schizophrenia is a chronic mental illness characterized by positive, negative and cognitive symptoms. Positive symptoms consist of hallucinations, delusions, disorganized behavior and disorganized speech. Negative symptoms consist of alogia, anhedonia, asociality, affective flattening and impaired attention and concentration. Cognitive symptoms consist of impairment in working memory and executive functioning. Schizophrenia presents in various symptom cluster other than typical of schizophrenia with positive and negative symptoms. Obsessive compulsive symptoms also occur frequently in patients with schizophrenia but they are not considered essential elements of schizophrenia. The presence of obsessive- compulsive symptoms in patients with schizophrenia offers another subtyping strategy including phenomenologic, neurobiologic treatment response and outcome characteristics. They also cause significant distress and anxiety leading to impairment in the social, occupational and interpersonal functioning.
\end{abstract}

Material \& Methods:

After obtaining the necessary approval the study was started with enrolling of 60 newly diagnosed cases of schizophrenia fulfilling the DSM-V criteria for the diagnosis of schizophrenia. The prevalence of obsessive-compulsive symptoms in the patients was assessed using the Yale- Brown Obsessive Compulsive Scale (Y-BOCS) the severity of positive and negative symptoms was assessed using the Positive And Negative Syndrome Scale (PANSS).

Results:

The prevalence of obsessive - compulsive symptoms in patients with schizophrenia was found to be $35 \%$. A significant corelation was found with PANSS Positive score, PANSS General score and composite score suggesting that patients of schizophrenia with OCS are more likely to have positive symptoms. The higher scores on Y-BOCS also had higher scores on PANSS suggesting that the severity of schizophrenia has direct relationship with presence of OCS.

Index Terms- OCD, Obsessive Compulsive Disorder, Schizophrenia

\section{INTRODUCTION}

Schizophrenia is a staggering issue which fits under the more extensive heading, psychosis, which is described by loss of contact with reality. Schizophrenia is a chronic severe mental illness that affects approximately $0.5 \%$ to $1.5 \%$ of total worldwide population, and the annual incidence rate averages between 0.5 and 5.0 per 10,000 people. ${ }^{1}$ Studies suggest that the prevalence of schizophrenia is found higher in men compare to female. Schizophrenia additionally generally begins prior among men. $^{2}$ Schizophrenia includes positive symptoms like hallucinations (hearing seen or feeling things that not there), delusions (fixed false beliefs or suspicions that are firmly held even when there is evidence to contrary) \& abnormal/ disorganized behavior (strange appearance, self-neglect, incoherent speech, wandering aimlessly or mumbling or laughing to self). ${ }^{2}$ It includes negative symptoms like alogia, anhedonia, affective flattening, asociality and impaired attention and concentration. Schizophrenia is the most puzzling and debilitating of all the psychiatric disorders and characterized by disordered cognition, including a "gain of function" in psychotic symptoms and loss of functions in specific cognitive functions such as working memory and declarative memory but without the progressive dementia that characterizes classical neurodegenerative disorders. It is perhaps the most dramatic and tragic manifestations of mental illness known to mankind. The consequences of the illness for the individual affected, his or her family, and society in general are devastating. ${ }^{3}$

Obsessive compulsive syndrome is a disorder of brain and behaviour which causes severe anxiety and distress in those who are affected. It involves both obsessions and compulsions that take a lot of time and causes significant impairment in functioning. These symptoms are clinically important for the management and prognosis. The obsessive compulsive symptoms can be of various kinds like contamination, sexual, religious, aggressive or somatic themes, with or without accompanying compulsions such as cleaning, checking, hoarding, repeating and arranging.

Obsessive compulsive symptoms in schizophrenia are well recognized and have been described in various forms as a part of schizophrenia but are a less-researched entity. There has also been a growing interest in finding more meaningful subtypes of schizophrenia than those described in both the classic and 
modern nomenclatures which assumes that schizophrenia is a heterogeneous disorder with diverse underlying neurobiologic mechanisms. The presence of obsessive-compulsive symptoms within the schizophrenia spectrum may offer yet another subtyping strategy, with discrete phenomenologic, neurobiologic treatment response and outcome characteristics.

\section{MATERIALS AND METHODS}

Study Design:

Our study was an observational cross-sectional study to study the obsessive - compulsive symptoms in patients of schizophrenia.

Sample Size 60 newly diagnosed cases of schizophrenia were taken to get $30 \%$ mean improvement with 14.4 SD and absolute precision of 5 at $99 \%$ confidence.

Site of Study:

The study sample was obtained from clinical departments IPD and OPD based patients in Dhiraj General Hospital (DGH), Smt. B K Shah Medical Institute and Research Centre (SBKS MI\&RC), Piparia. Dhiraj General Hospital covers major population of Piparia village, Vadodara and nearby districts of Gujarat and Madhya Pradesh.

\section{INCLUSION CRITERIA}

1. Patients ready to participate and give a written, informed consent.

2. Newly diagnosed cases of schizophrenia fulfilling the DSM-V criteria for diagnosis of schizophrenia.

3. Patients aged between 18-65 years of both gender.

\section{EXCLUSION CRITERIA}

1. Patients not willing to give written consent.

2. Patients with debilitating medical condition.

3. Patients with delirium or dementia.

4. Patients with intellectual disabilities.

5. Acutely agitated patients or patients not able to perform study related assessments.

\section{METHODOLOGY}

The study was aimed to study the prevalence of obsessivecompulsive symptoms in patients with schizophrenia and their co-relation with the positive and negative symptoms of schizophrenia. This was an observational study. Study was started after obtaining permissions from Sumandeep Vidyapeeth Institutional Ethics Committee (SVIEC). The study was started and the patients were enrolled after the approval of the Sumandeep Vidyapeeth Ethics Committee. The patients were explained about the interview and after they are willing to participate in the study, a written, informed consent was taken from the patient in the language of their choice. After the consent, the patients were assessed for inclusion in the study, using a structured clinical interview for diagnosis (SCID) of schizophrenia. All demographic details of the patient were recorded using the Case Report Form (CRF).

The prevalence of obsessive-compulsive symptoms will be assessed using the Yale- Brown Obsessive Compulsive Scale (Y-
BOCS). The severity of positive and negative symptoms will be assessed by Positive And Negative Syndrome Scale (PANSS).

\section{RESULT AND OBSERVATON}

The sample consisted of 60 patients of newly diagnosed cases of Schizophrenia. Various socio-demographic details were compared using appropriate statistical tools. Various tests like Chi Square test, independent t-test and Pearson's correlation coefficient has been used to find correlation between many variables.

\section{Socio-demographic details:}

In present study majority of the patients are of young age group and mean age of all enrolled patients were $32.10+/-10$ years and similarly mean age of male patients was 31,50 +/- 9.37 years whereas mean age of female patient was $32.79+/-9.52$ years.

In the study amongst 60 participants, $32(53.33 \%)$ were male and $28(46.67 \%)$ were female. Out of the 60 patients enrolled in the study, $31.7 \%$ belonged to the urban population and $68.3 \%$ belonged to the rural population. Out of all enrolled patients we have found that unemployment status was higher, i.e. $40.00 \%$ were unemployed followed by $33.33 \%$ were employed, $3.33 \%$ were businessman and $23.33 \%$ were students. In present study it was noted that half of the patients were married, i.e. $53.33 \%$ followed by $33.33 \%$ were unmarried, $5.00 \%$ were widow \& separated and $1.67 \%$ was widower \& divorced respectively. In the study, $6.6 \%$ of population belonged to the upper class, $36.6 \%$ of population belonged to the upper middle class, $43.3 \%$ belonged to the lower middle class and $6.6 \%$ to the lower socioeconomic class.

The co-relation of OCS with demographic variables like age, gender, residence, marital status, employment status and socioeconomic status was assessed and the p-value was 0.074, 0.914, 0.051, 0.213, 0.078 and 0.491 respectively using Chi Square test and no significant correlation was found between the age, gender, residence, marital status, employment status, socioeconomic status with the prevalence of OCS.

\section{Table 1: Prevalence of Obsessive Compulsive symptoms in} Study Participants

\begin{tabular}{|l|c|}
\hline Y-BOCS score & N (\%) \\
\hline Subclinical OCS (0-7) & $39(65 \%)$ \\
\hline Mild OCS (8-15) & $7(11.7 \%)$ \\
\hline Moderate OCS (16-23) & $4(6.6 \%)$ \\
\hline Severe OCS (24-31) & $7(11.7 \%)$ \\
\hline Extremely Severe OCS (32-40) & $3(5.0 \%)$ \\
\hline Prevalence of OCS & $21(35 \%)$ \\
\hline
\end{tabular}

A Y-BOCS score greater than 7 is considered as having OC symptoms. So, $35 \%$ of study population was found to be having OC symptoms. According to the present study, the prevalence of 
OC symptoms in patients with schizophrenia is $35 \%$. In this study it was observed that $11.7 \%$ participants were having mild OC symptoms, $6.6 \%$ were having moderate OC symptoms, $11.7 \%$ were having severe OC symptoms and $5 \%$ of participants were having extremely severe OC symptoms.

Table 2: Co-relation of Obsessive-Compulsive Symptoms with clinical variables of Study Participants

\begin{tabular}{|c|c|c|c|c|c|}
\hline & OCS & Mean & SD & $\mathrm{t}$ value & $\mathrm{p}$ value \\
\hline \multirow{2}{*}{$\begin{array}{c}\text { Age of onset } \\
\text { of illness }\end{array}$} & Yes & 32.10 & 8.944 & \multirow[t]{2}{*}{1.674} & \multirow[t]{2}{*}{.100} \\
\hline & No & 27.92 & 9.345 & & \\
\hline \multirow{2}{*}{$\begin{array}{l}\text { Duration of } \\
\text { illness }\end{array}$} & Yes & 2.925 & 1.644 & \multirow[t]{2}{*}{0.721} & \multirow[t]{2}{*}{.474} \\
\hline & No & 2.581 & 1.809 & & \\
\hline \multirow{2}{*}{$\begin{array}{l}\text { PANNS } \\
\text { Positive }\end{array}$} & Yes & 18.29 & 4.849 & \multirow[t]{2}{*}{-1.986} & \multirow[t]{2}{*}{.048} \\
\hline & No & 21.03 & 5.224 & & \\
\hline \multirow{2}{*}{$\begin{array}{l}\text { PANNS } \\
\text { Negative }\end{array}$} & Yes & 29.48 & 6.055 & \multirow[t]{2}{*}{1.506} & \multirow[t]{2}{*}{.138} \\
\hline & No & 25.74 & 10.427 & & \\
\hline \multirow{2}{*}{$\begin{array}{l}\text { PANNS } \\
\text { General }\end{array}$} & Yes & 48.48 & 11.860 & \multirow[t]{2}{*}{2.620} & \multirow[t]{2}{*}{.011} \\
\hline & No & 41.85 & 7.707 & & \\
\hline \multirow{2}{*}{$\begin{array}{l}\text { Composite } \\
\text { score }\end{array}$} & Yes & -11.19 & 6.969 & \multirow[t]{2}{*}{-2.164} & \multirow[t]{2}{*}{.035} \\
\hline & No & -4.72 & 12.680 & & \\
\hline \multirow[t]{2}{*}{ PANNS Total } & Yes & 96.24 & 17.660 & \multirow[t]{2}{*}{1.632} & \multirow[t]{2}{*}{.108} \\
\hline & No & 88.62 & 17.044 & & \\
\hline
\end{tabular}

In the present study the co-relation of OCS with clinical variables like age of onset of illness, duration of illness, PANSS Positive score, PANSS Negative score, PANSS General score, Composite score and PANSS Total was assessed and the p-value was 0100 , $0.474,0.048,0.138,0.011,0.035,0.108$ respectively respectively using independent t-test and no significant correlation was found between age of onset of illness, duration of illness, PANSS Negative score, PANSS Total score with the prevalence of OCS. A significant correlation was found between PANSS Positive score, PANSS General score and Composite score and the prevalence of OCS.

Table 3: Co-relation of Y-BOCS with PANSS using Pearson's Correlation Coefficient

\begin{tabular}{|l|c|c|}
\hline & Correlation coefficient & p-value \\
\hline PANNS Total & 0.366 & 0.004 \\
\hline PANNS General & 0.309 & 0.016 \\
\hline
\end{tabular}

The Y-BOCS score is co-related with the PANSS Total score and PANSS General score using Pearson's correlation coefficient and the p-value was found to be 0.004 and 0.016 , both of which are statistically significant. It was noted that the higher scores on YBOCS also had higher scores on PANSS Total score and PANSS General Score.

\section{DISCUSSION}

In the present study total 60 patients of schizophrenia were enrolled ranging from 18 to 65 years, out of them $53.3 \%$ were male and $46.7 \%$ were female, $68.3 \%$ belonged to rural population and $31.7 \%$ belonged to urban population, $53.30 \%$ of patients were married followed by $33.30 \%$ patients were single, $40.00 \%$ were unemployed followed by $31.33 \%$ were employed, $3.33 \%$ were businessman and $23.33 \%$ were students, $6.6 \%$ of population belonged to the upper class, $36.6 \%$ of population belonged to the upper middle class, $43.3 \%$ belonged to the lower middle class and $6.6 \%$ to the lower socio-economic class. The majority of the patients are of young age group and mean age of all enrolled patients were $32.10+/-10$ years and similarly mean age of male patients was $31.50+/-9.37$ years whereas mean age of female patient was $32.79+/-9.52$ years.

Obsessive-compulsive symptoms (OCS) frequently occur in a substantial proportion of patients in schizophrenia though a wide variation in prevalence is noted. Worldwide prevalence of OCS is ranging from $1.1 \%$ to $50 \%$ where as in India prevalence of OCS is found in $10.57 \%$ to $24.0 \%$ of population. ${ }^{4}$ In the present study, the prevalence of OCS is $35 \%$ in patients with schizophrenia.

In this study we aimed at assessing the relationship of positive and negative symptoms with the obsessive-compulsive symptoms. The co-relation of positive and negative symptoms and OCS were calculated and it was found that the mean value of PANSS Positive score in patients with OCS was 18.29 with SD of 4.84 and without OCS was 21.03 with SD of 5.22 and using independent t-test the p-value was observed to be 0.048 , the mean value of PANSS Negative score in patients with OCS was 29.48 with SD of 6.05 and without OCS was 29.74 with SD of 10.42 and the p-value was observed to be 0.138 , the mean value of PANSS General score in patients with OCS was 48.48 with SD of 11.86 and in patients without OCS was 41.85 with SD of 7.70 and the p-value was observed to be 0.011 and the mean value of PANSS Total score in patients with OCS was 96.84 with SD of 17.66 and without OCS was 88.62 with SD of 17.04 and the p-value was observed to be 0.108 . No significant co-relation of PANSS Negative score and PANSS Total score with OC symptoms were observed and significant co-relation was noted between PANSS Positive score and PANSS General score with OC symptoms.

The study concluded that patients of schizophrenia with obsessive- compulsive symptoms were more likely to have positive symptoms and more general psychopathology and no relationship has been observed between negative symptoms and obsessive-compulsive symptoms. Various other studies have been conducted to study the relationship of obsessivecompulsive symptoms and the positive and negative symptoms of schizophrenia. Various studies found differences in clinical characteristics of patients with schizophrenia and co-morbid OC symptoms compared to schizophrenia alone. The pattern of the study is highly variable. Some studies reported less severe positive and negative symptoms, others reported no differences and others more severe positive and negative symptoms in this population.

A study conducted by M. Byerly et al. ${ }^{5}$ found that the mean value of PANSS Positive score in patients with OCS was 16 with SD of 5.4 and without OCS was 15.3 with SD of 5.7 and using independent $\mathrm{t}$-test the $\mathrm{p}$-value was observed to be 0.62 , the mean 
value of PANSS Negative score in patients with OCS was 17.3 with SD of 5.9 and without OCS was 18.8 with SD of 6.5 and the p-value was observed to be 0.39 , the mean value of PANSS General score in patients with OCS was 35.2 with SD of 7.8 and in patients without OCS was 34.8 with SD of 7.4 and the p-value was observed to be 0.82 and the mean value of PANSS Total score in patients with OCS was 68.6 with SD of 15.6 and without OCS was 68.9 with SD of 16 and the p-value was observed to be 0.96 . No statistically significant difference were found for any of the symptoms in the study establishing that there is no relationship between obsessive-compulsive symptoms and the symptoms of schizophrenia.

Hermom et $\mathrm{al}^{6}$. also calculated the co-relation between positive and negative symptoms and obsessive-compulsive symptoms using the Spearman's Rho and no significant co-relation was observed between the symptoms of schizophrenia and obsessivecompulsive symptoms. Similar findings are reported by Berman et al. (1998) and Chakraborty et al. (2004). Lysaker et al. (2000) reported more positive symptoms in patients with obsessivecompulsive symptoms and Nechmand et al. (2003) ${ }^{7}$ reported more negative symptoms in schizophrenia with obsessivecompulsive symptoms.

Also, Fabisch et al. ${ }^{8}$ showed that patients with schizophrenia who had OCS were more likely to have negative symptoms.

Vast variety has been observed in the results establishing the relationship of obsessive-compulsive symptoms with the positive and negative symptoms of schizophrenia owing to the differences in the diagnostic criteria, method of evaluation, chronicity of the illness, in-patients versus outpatients, early versus chronic stage of illness, duration of illness, exacerbated versus continuous deteriorating course. So, further evaluation should be beneficial in concluding the relationship of obsessive-compulsive symptoms and the symptoms of schizophrenia.

In the present study, the Y-BOCS score is co-related with the PANSS Total score and PANSS General score using Pearson's correlation coefficient and the values are 0.366 and 0.309 and the p-value was found to be 0.004 and 0.016 respectively, both of which are statistically significant. It was noted that the higher scores on Y-BOCS also had higher scores on PANSS Total score and PANSS General score. Hence the findings suggest that the severity of schizophrenia has direct relationship with the presence of obsessive-compulsive symptoms. According to a study conducted by Bulent Kayahan et al. ${ }^{9}$, the Y-BOCS score was co-related with the PANSS Positive score, PANSS General score and PANSS Total score using Pearson's correlation coefficient and the values were $0.349,0.404,0.370$ respectively and the p-value was less than 0.005 for all the scores which was statistically significantly. The study concluded that the obsessivecompulsive symptoms were found significantly correlated to total PANSS score, PANSS positive symptoms, PANSS General score and not significantly correlated to PANSS Negative symptoms. These findings are consistent with findings of the study conducted by Lysaker et al. ${ }^{3}$ The results support the hypothesis that obsessive-compulsive symptoms in schizophrenia reflect the presence of two co-morbidities which may functionally augment and exacerbate one another. The difference between the studies maybe due to the difference in diagnostic criteria, method of evaluation, chronicity of the illness, in-patients versus outpatients, early versus chronic stage of illness, duration of illness, exacerbated versus continuous deteriorating course.

\section{V.CONCLUSION}

1. We may conclude that the existence of obsessive-compulsive symptoms is a relatively frequent finding in patients with schizophrenia. The prevalence of obsessive- compulsive symptoms is high in patients with schizophrenia.

2. Patients of schizophrenia with obsessive- compulsive symptoms had more severe positive symptoms and general psychopathology symptoms and no relationship with the negative symptoms.

3. Severity of schizophrenia has direct relationship with the presence of obsessive-compulsive symptoms.

\section{REFERENCES}

[1] Alshowkan, A., Curtis, J. \& White, Y. 2012, 'Quality of life for people with schizophrenia: a literature review, The Arab Journal of Psychiatry, vol. 23, no.2, pp. 122-131

[2] Schizophrenia Fact Sheet 397" WHO September 2015. Reviewed April 2016.

[3] Lysaker PH, Marks KA, Picone JB, Rollins AL, Fastenau PS, Bond GR. Obsessive and compulsive symptoms in schizophrenia. Clinical and Neurocognitive correlates. J. Nerv. Ment. Dis. 2000; 188: 78-83.

[4] Obsessive-Compulsive symptoms in patients with Schizophrenia attending tertiary care centre in South India, Punya Mulky, Vol 2 | Isue 4 | OctoberDecember $\mid 2015$.

[5] M.Byerly et al. 2005. Obsessive - compulsive symptoms in schizophrenia Frequency and clinical features. 76; 309 - 316.

[6] SmitaHemrom, Pushpa, Divya Prasad, MasroorJahan, Amool R. Singh, D.K. Kenswar. Hemrom, "Prevalence of obsessive compulsive symptoms among patients with schizophrenia"; Industrial psychiatry journal 2009;18(2):77-80

[7] Nechmad A, Ratzoni F, Poyurovsky M, Meged S, Avidan G, Fuchs C, et al 2003. Obsessive - compulsive disorder in adolescent schizophrenic patients. Am J Psychiatry 160:1002 - 1004.

[8] Fabisch K, Fabisch H, Langs G, Weiselmann G, Zapotoczky HG. 1997 Obsessive-compulsive symptoms in schizophrenia. Schizophr Res 24:17

[9] Obsessive-compulsive symptoms in schizophrenia: Prevalence and clinical correlates, BULENT KAYAHAN, Psychiatry and Clinical Neurosciences (2005), 59, 291-295. 


\section{AUTHORS}

First Author -

Dr. Dhun Patel,

$3^{\text {rd }}$ year resident in M.D.Psychiatry, SBKS MI \& RC.

Email-pateldhun07@gmail.com

Second Author -

Dr. Lakhan Kataria,

M.D. Psychiatry, SBKS MI \& RC.

Email-drlakhankataria@gmail.com

\section{Third Author -}

Dr. Suhani Desai,

M.D. Psychiatry.

Email-suhani.capricon89@gmail.com

\section{Fourth Author --}

Dr. Kinnari Trivedi

$3^{\text {rd }}$ year resident in M.D.Psychiatry, SBKS MI \& RC.

Email-drkinnaritrivedi@gmail.com

\section{Correspondence Author -}

Dr. Dhun Patel,

Email-pateldhun07@gmail.com, pateldhun7@gmail.com,

Contact number- +919662092227 